\title{
Nurses' Awareness of Infection Control Measures, and the Role and Effect in Patient and Family Education
}

\author{
Sahar Hammoud, (BSc,MSc) \\ Bassam Ghazi, (MD) \\ Mohamad Nassredine, (PhD) \\ Lebanese University/Faculty of Sciences, Lebanon \\ Mohamad Abou Haidar, (DPT,PhD) \\ Lebanese University/Faculty of Public Health, Lebanon
}

doi: 10.19044/esj.2017.v13n27p59 URL:http://dx.doi.org/10.19044/esj.2017.v13n27p59

\begin{abstract}
Background: Healthcare Associated Infections are serious problems in healthcare sector that threatened patient safety since decades till present. The proper adherence to infection control measures is a major factor in reducing these infections. Objectives: The objectives of this study were, to assess the level of nurses' awareness with infection control measures in Lebanon and to determine its role in implementing patient and family education. Methodology and Results: A quantitative study was done. Questionnaires were distributed over 260 nurses, and 260 patients and family members at four well known hospitals in Lebanon. The study showed a high level of infection control awareness (81.57 \%) among Lebanese nurses, and showed that Lebanese hospitals are training their nurses on IC topics (99.1\% of nurses were trained). As for patients and family members' education, the JCI accredited hospital showed the highest level of education on infection control measures $(58.3 \%)$. Conclusion and Recommendations: The study showed that nurses who had a high awareness in infection control educated more their patients and family members on these measures than nurses with low awareness. (64.1\% and $42.1 \%$ when educating on respiratory hygiene, $\mathrm{p}=0.013,86.2 \%$ and $57.9 \%$ when educating on usage of PPE, $\mathrm{p}=0.000,89.2$ $\%$ and $68.4 \%$ when educating on the reason for isolation, $\mathrm{p}=0.001)$. The major recommendations were to enhance the culture that believes in the partnership between patients, their families and healthcare providers at the hospitals level, and to include a new standard in the Lebanese Accreditation Standards that requires educating patients and family members on infection control measures at the Ministry of Public Health level.
\end{abstract}


Keywords: Infection Control - Nurses - Patients - Education - Accreditation Standards)

\section{Introduction}

The most serious problem patients are facing during their hospital stay from decades till present is Healthcare Associated Infections (HAI). Based on a study that was conducted by the Center for Disease Control and Prevention (CDC) in the United States (U.S.), we can say that at any given time, one of 25 hospitalized patients at the U.S. acquires a HAI. This means that every year there are 650,000 patients with a HAI. (Agency for health care research and quality (AHRQ), 2015). In spite of the improvement and evolution in healthcare sector in general and especially patient safety during the last decades, HAI are still acquired and growing inside hospitals $(\mathrm{H}$. Seale, A. Chughtai, R. Kaur, P. Crowe, L. Phillipson, Y. Novytska, J. Travaglia, 2015). These infections are affected by the noncompliance, and the poor adherence to Infection Control (IC) measures (standard precautions, Transmission-based precautions, IC policies) that aim at reducing the transmission of HAI. The level of compliance with IC measures differs from one Healthcare Worker (HCW) to another, and the level of HCW knowledge and awareness of IC measures also differs across healthcare facilities and countries, depending on the level of trainings being conducted by the IC, the financial state of the facility and the overall country, and commitment of the general administration (K. Vaz, D. McGrowder, R. Alexander-Lindo, L. Gordon, L. Brown, R. Irving, 2010). For further improvement many suggestions emphasized on empowering patients by engaging and involving them in the process of care, to perform as a dynamic part in their care process. Empowering the patients' means allowing them to achieve the information, abilities and the approach they need to make decisions and participate in their own process of care by educating and encouraging them to participate in all aspects. Later on emphasizes were on engaging the patient in many IC related measures and aspects after proving that this empowerment through education and participation will lead to reducing the risk of HAI and thus providing a safer care $(\mathrm{H}$. Seale, A. Chughtai, R. Kaur, P. Crowe, L. Phillipson, Y. Novytska, J. Travaglia, 2015).

Lebanon has started his first accreditation project in 2001 based on international standards. Later on, in 2006 the Ministry of Public Health has signed up an assistance contract with the French Health Authority (HAS) to advance the hospital accreditation program. Two chapters were added to the Accreditation manual so they became forty chapters. IC chapter is one of these chapters, and according to this chapter, an annual education program shall exist to all hospital staff (W.Ammar, R.Wakim, I.Hajj, 2007). So these 
trainings shall increase nurses' awareness in IC. And having nurses who are highly aware of these measures will enhance the proper education for the patients and their families on IC measures that will help in minimizing the risk of acquiring a HAI and thus improving the quality of patient care and life. No studies were conducted in Lebanon to determine the level of nurses' awareness of IC measures and determined the effect or the role of this awareness in educating the patients and their families on these IC measures, so this study was conducted to assess nurses' awareness level of IC measures, and to determine the role of this awareness in implementing patient and family education.

\section{Methods}

Based on the literature, and after the thorough research that was done on studies that were conducted through years to assess the level of Nurses and HCW awareness on IC measures, it was found that most of these studies used quantitative methods using questionnaires in order to assess the IC awareness level. The nine studies that were mentioned in the literature review of this study also used quantitative methods, (C. Regina, A. Molassiotis, C. Eunice, C. Virene, H. Bedcy, L. Chit-Ying, L. Pauline, S. Frances, Y. Ivy, 2002), (M. Askarian, K. Aramesh, C. John Palenik, 2002), (A. Shah Salehi, P. Garner, 2010), (K. Vaz, D. McGrowder, R. AlexanderLindo, L. Gordon, L. Brown, R. Irving, 2010), (A. Elgilany, K. Badawy, B. Sarraf, 2012), (A. A Charya, J. Khandekaar, A. Sharma, T. HR, A.Kataria, 2013), (A. Tenna, E. Stenehjem, L. Margoles. E. Kacha. H. Blumberg, R. Kempker, 2013), (S. ALZahrani, F. Al-Army, M. Ghonaim, O. Abo-Salem, 2013), (S. Mudedla, K. Reddy, M. Sowribala, W. Tej, 2014). On the other hand, all the studies that were mentioned in the study literature and were done to assess patients and family members' knowledge of IC measures, the education acquired by them about IC, the level of patients and families' understanding and knowledge of HAI, their way of transmission, and how to prevent them, (P. Miller, B. Farr, 1989), (R. Abbate, G. Giuseppe, P. Marinelli, I. Angelill, 2008), (M. Macleo, L. Shields, E. Owen, 2008), (A. Sengupta, C. Rand, T. Perl, A. Milstone, 2010), (M. Atti, A. Tozzi, G. Ciliento, M. Pomponi, S. Rinaldi, M. Raponi, 2011), (S. Pan, K. Tien, I. Hung, Y. Lin, Y. Yang, M. Yang, M. Wang, S. Chang, 2013), (I. Ocran, D. Tagoe, 2014), (H. Seale, Y. Novytska, J. Gallard, R. Kaur, 2015), (P. Chittick, S. Koppisetty, L. Lombardo, A. Vadhavana, A. Solanki, K. Cumming, V. Agboto, C. Karl, J. Band, 2016), (F. Bellissimo-Rodrigues, D. Pires, W. Zingg, D. Pittet, 2016), also used quantitative methods to complete their studies. So based on the research and literature, a quantitative method was selected to complete this study. 
Two questionnaires were prepared in consultation with professionals at IC Departments. Before distributing questionnaires a quantitative pilot study was done on a selective group of nurses, patients and family members. After that minimal changes were done to both questionnaires. Before distributing the questionnaires on participants, the purpose of the study was explained to each participant and confidentiality of information was assured. A consent form was signed by each participant.

Sample: Self-administered questionnaires were distributed to nurses at four well known hospitals in Lebanon (a university public hospital, a university private hospital, a non-university private Joint Commission International (JCI) accredited hospital and a non-university private hospital), after taking Institutional Review Board (IRB) or top management approvals according to each hospital internal rules and regulations. 260 questionnaires were distributed on nurses in all in-patient units (65 questionnaires for each hospital). At the same time, another questionnaires were distributed to patients and family members at the same four mentioned hospitals (260 questionnaires), participants were patients who were admitted as inpatients and have been for more than 24 hours at the hospital, and their family members presented at that time.

Questionnaire design: Nurses' questionnaire, a 21-item selfadministered questionnaire composed of four parts: The first part included socio-demographic information (gender, age, job tittle, educational level, number of service years at the organization, name of hospital and the unit). The second part consisted of fourteen questions to assess the awareness of nurses regarding IC in seven areas: HAI, Biomedical waste handling, Linen handling, Handling / disposal of sharps, Hand hygiene, Blood-borne diseases, Personal Protective Equipment (PPE), Additional safety measures. The third part of was about nurses' education and trainings on IC measures including the training topics. The fourth part aimed at determining whether nurses were educating their patients and family members on IC measures (including the topics). Patients and family members' questionnaire consisted of two parts; the first part was demographics part (gender, age, educational level, name of hospital and the unit). The second part consisted of nine questions to determine whether patients and family members were being educated by nurses on general IC measures such as, HAI and their risks, hand hygiene, respiratory hygiene, reason of isolation and the usage of PPE (for isolated patients).

Construction of IC Awareness Score: The survey included 14 elements of IC of which the nurses should be fully aware. Individuals who answered correctly all 14 elements were assigned a score of 100 while those who answered none were assigned a score of zero. Additionally, an IC awareness variable with two categories was constructed as follows; 
respondents who answered correctly 10 elements or more were classified as having high awareness level (a corresponding score above 70 over 100) while those who answered correctly 9 elements or less were classified as having low awareness level (a score that is below 70 over 100).

Statistical Analysis: For univariate analysis a simple summary of numbers and their percent was used for categorical variables and mean and standard deviation was used for numeric continuous variables. For bivariate analysis, the statistical test used was Chi-square, because both the independent and the dependent variables were nominal. The dataset included one numeric continuous variable which was the composite score of staff awareness about different IC measures. This score had a minimum of 0 and a maximum of 100. For this score, mean comparison was used to test for effects of independent variables on staff awareness (t-test for variables with two categories and One Way Analysis of Variance -ANOVA- for variables with more than one attribute). Alpha level of 0.05 was used as the cutoff point of statistical significance in all statistical tests. SPSS version 18 was used to run the statistical analysis.

\section{Results:}

The response rate in filling the questionnaires was $83.46 \%$ for nurses (217) and $85.77 \%$ for patients and family members (223).

\subsection{Nurses' Descriptive Analysis Nurses' Awareness of IC Measures}

Concerning nurses IC awareness level, $81.57 \%$ of nurses had a high awareness and $18.43 \%$ had a low awareness. $63.6 \%$ of nurses were aware of HAI, 94.9\% were aware of handling biomedical wastes, $97.2 \%$ were aware of handling infected linen, $87.1 \%$ were aware of handling sharps, 44.2 $\%$ were aware of hand hygiene, $13.8 \%$ were aware of blood borne diseases, $54.4 \%$ were aware of additional safety measures and $71.9 \%$ were aware about the usage of PPE (Table 1).

\section{Nurses' Trainings on IC Measures}

As for nurses' training on IC Measures, 99.1\% of the nurses attended trainings on IC measures at their current hospital and $61.9 \%$ of them attended these trainings 3 months ago. 90.2 \% were trained on Hand Hygiene, $80.5 \%$ were trained on Usage of PPE, 77.2\% on standard precautions, $74.9 \%$ on Isolation and finally $58.1 \%$ were trained on managing hospitals spills. 


\section{Nurses' Education of Patients and Family Members on IC Measures}

$94.5 \%$ of nurse stated that they did educate their patients and family members on IC measures. Out of these $94.5 \%, 90.7 \%$ stated that they did educate their patients and family members on Hand Hygiene, $60 \%$ educated them on Respiratory Hygiene, $85.4 \%$ informed them the reason for placing them in Isolation, $81 \%$ educated them on Usage of PPE and $34.6 \%$ educated them on HAI and the risk of acquiring it. $87.3 \%$ of nurses who educated their patients and family members, educated them upon admission and during hospital stay.

\section{Patients and Family Members' Descriptive Analysis}

Coming to patients and family member's questionnaire, out of the respondents who were 223 as a whole, $52 \%$ were patients and $48 \%$ were family members.

\subsection{Patients and Family Members' Education on IC Measures}

Among the respondents, 55.2\% stated that they were educated at least on one IC measure or topic, while $44.8 \%$ were not. $34.5 \%$ of respondents stated that they were educated on HAI and the risk of acquiring a HAI in hospitals. $21.1 \%$ were educated on respiratory hygiene/cough etiquette, $29.1 \%$ were provided with brochures regarding hand hygiene and/or respiratory hygiene. Concerning patient isolation status, $15.2 \%$ stated that they were in isolation at the time of filling the questionnaires, among these who were on isolation, $79.4 \%$ were informed about the reason for placing them in isolation and $82.4 \%$ were educated on the usage of PPE. As for the time of education, $49.8 \%$ stated that they were educated upon admission and during their hospital stay.

\subsection{Nurses Bivariate Analysis:}

\section{IC Awareness of Nurses by their Demographic Characteristics}

The percentage of nurses who had high awareness in IC measures did not differ by respondent's gender, age or years of service at current hospital. When comparing across Job titles, Head Nurses (HN) had the highest awareness level followed by Registered Nurses (RN), then Practical Nurses $(\mathrm{PN}), 96 \%, 84.7 \%$ and $68.1 \%$ respectively $(\mathrm{p}=0.004)$. As for the effect of respondents' educational level, the highest IC awareness level was among respondents' with Master's degree and the lowest was among respondents' with TS or lower degree $(94.1 \%$, and $66.7 \%$ respectively, $\mathrm{p}=0.001)$ (Table 2). 


\section{Nurses' Educational Activities for Patients and Family Members by} Nurses' Level of IC Awareness

The study showed also that nurses with high awareness level stated a higher rate in educating their patients/family members than nurses with low awareness who stated a lower rate as follows, when educating on respiratory hygiene $(64.1 \%$ and $42.1 \%, \mathrm{p}=0.013)$, reason for isolation $(89.2 \%$ and $68.4 \%, \mathrm{p}=0.001)$ and usage of PPE (86.2 \% and $57.9 \%, \mathrm{p}=0.000)$ (Table 3).

\subsection{Patient and Family Member's Bivariate Analysis:}

\subsubsection{Stated Education of Patients and Family Members on HAI by their Demographic Characteristics}

The percentage of patients or family members who stated they were educated on HAIs did not differ by respondent's status, age, or gender. The stated education rate was the highest by respondents in the JCI accredited hospital $(58.3 \%, \mathrm{p}=0.000)$.

\subsubsection{Hand Hygiene / Respiratory Hygiene Brochures Distribution by Hospitals}

Concerning the percentage of distribution of hand hygiene and / or respiratory hygiene brochures across hospitals, the highest rate of distribution was among the JCI accredited hospital also $(81.7 \%, \mathrm{p}=0.000)$ (Figure 1).

\section{Discussion:}

\subsection{Nurses' Descriptive Analysis}

Nurses' Awareness of IC Measures

This study measured the awareness level of nurses in various IC measures, where $81.57 \%$ of nurses had a high awareness and $18.43 \%$ had a low awareness, these results seems to be much better than others compared to a study that was conducted in Kingdom Of Saudi Arabia (KSA) in 2012 where only $63 \%$ of participants were aware of IC measures (S. ALZahrani, F. Al-Army, M. Ghonaim, O. Abo-Salem, 2013). Going through IC topics, $63.6 \%$ of nurses were aware of HAI, while in a similar study that was conducted in Ghana in 2012, $88.7 \%$ of HCW were knowledgeable of HAI (I. Ocran, D. Tagoe, 2014). A low level of awareness in hand hygiene was observed where $44.2 \%$ of nurses were aware, while $71.9 \%$ were aware of usage of PPE. Comparing this study results to a study that was done in Taiwan in 2013 a higher level of awareness in hand hygiene was found where $89 \%$ of their HCW were aware, but similar level of awareness in usage of PPE was found compared to this study (69\%), this low level of awareness in hand hygiene (44.2 \%) maybe due to nurses' negligence, work 
overload, or due problems related to management commitment, as the lack of or the inaccessibility to hand washing facilities or hand antiseptics, and not due to lack of training because as we mentioned above $90 \%$ of the nurses stated that they did attend training on hand hygiene. At the same time the study showed a high level of awareness in handling biomedical wastes, handling linen and handling/disposal of sharps $(94.9 \%, 97.2 \%$ and $87.1 \%$ respectively), while the opposite was found in a study that was conducted in India (Hyderabad) in 2012 where their HCW were only somewhat knowledgeable regarding biomedical wastes management and sharps disposal (S. Mudedla, K. Reddy, M. Sowribala, W. Tej, 2014). This high level of awareness found in this study regarding these topics can be explained by the continuous education that was being done in these Lebanese hospitals where $99.1 \%$ of nurses stated that they were trained on IC Measures.

\section{Nurses' Trainings on IC Measures}

The study results about nurses' trainings on IC measures $(99.1 \%$ of nurses stated that they were trained) were similar to a study that was conducted in 2012 in KSA which showed that all of the participants attended training on IC policies (S. ALZahrani, F. Al-Army, M. Ghonaim, O. AboSalem, 2013). These trainings lead to a high IC general awareness level among Lebanese nurses where $81.57 \%$ had a high awareness level as mentioned above.

\subsection{Patients and Family Members' Descriptive Analysis Patients and Family Members' Education on IC Measures}

$34.5 \%$ of patients and family members in this study mentioned that they were educated by nurses on HAI and the risk of acquiring a HAI, while in a similar study that was conducted in 2006 in Italy only $15.1 \%$ of the patients mentioned that they were informed by HCW about HAI (R. Abbate, G. Giuseppe, P. Marinelli, I. Angelill, 2008). And 22.3\% of patients in another study that was conducted in Australia in 2013 mentioned that they were informed about HAI by HCW (H. Seale, Y. Novytska, J. Gallard, R. Kaur, 2015). These low level of education on HAI that appeared in the literature supports the results of this study, although these results are being better but this also showed that Lebanese nurses are not highly educating their patients/family members on HAI. The reason behind this low level of education, may be due to the lack of efforts from Lebanese hospitals to explain to their staff the importance of patient education on IC measures, and due to the culture itself that does not really believe in the partnership between patients, their families and Healthcare Providers (HCPs). Also it would be due to the lack of such standard in the Lebanese accreditation 
standards that requests from hospitals to educate their patients and family members on IC measures.

\subsection{Nurses Bivariate Analysis:}

\section{IC Awareness of Nurses by their Demographic Characteristics}

This study showed a significant difference of awareness level when respondents were compared across Job titles $(\mathrm{p}=0.004)$, where HN had the highest awareness level while PN had the lowest awareness level, (96\% and $68.9 \%$ respectively), also this study showed a significant difference of awareness level when respondents were compared across educational level ( $p=0.001)$ where the highest IC awareness level was among respondents' with Master's degree and the lowest was among respondents' with TS or lower degree (94.1\% versus $66.7 \%$ respectively). These results are supported by the results of a study that was done in Egypt in 2010 where the knowledge of HCPs of standards precautions and IC differed also by respondent's Job title and educational level (A. Elgilany, K. Badawy, B. Sarraf, 2012).

\section{Nurses' Educational Activities for Patients and Family Members by Nurses' Level of IC Awareness}

When educating on Respiratory hygiene, nurses with high awareness level stated a higher rate in educating their patients/family members than nurses with low awareness $(64.1 \%$ and $42.1 \% \mathrm{p}=0.013)$, this was also the case when educating on Reason for isolation $(89.2 \%$ and $68.4 \% \mathrm{p}=0.001)$ and usage of PPE (86.2\% and $57.9 \% \mathrm{p}=0.000)$.

\subsection{Patient and Family Member's Bivariate Analysis: Stated Education of Patients and Family Members on HAI by their Demographic Characteristics}

This study showed also a significant difference in education rate of patients and family members (by their statement) on HAI among different nursing units $(\mathrm{p}=0.011$, Figure 2$)$ where Intensive Care Unit (ICU) patients/family members' stated the highest rate of education $(55.6 \%)$, this can be explained because ICU is a high risk area where critical patients are admitted, for that patients/family members in such area are being educated more than other patients in regular floors. Also a very significant difference was found in education rate among different hospitals $(\mathrm{p}=0.000)$, where JCI accredited hospital appeared to be the most hospital in educating its patients on HAI $(58.3 \%)$. 


\section{Hand Hygiene / Respiratory Hygiene Brochures Distribution by Hospitals}

A very significant difference was found also in the rate of distribution of brochures regarding hand hygiene and / or respiratory hygiene to patients and family members among different hospitals $(\mathrm{p}=0.000)$, where the JCI accredited hospital appeared to be the most hospital in providing these brochures $(81.7 \%)$.

This high rate of education and distribution of brochures can be explained because in JCI standards (fifth edition) there is a standard regarding Patient and Family members education on IC measures which requires from the hospital to provide education on infection prevention and control practices to staff, physicians, patients, families and other caregivers when indicated by their involvement in care. It also requests to encourage Patients and families to participate in the implementation and use of infection prevention and control practices in the hospital. "Standard PCI.11" (Jiont Commission International Standards for Hospitals, Fifth Edition, 2014).

This study showed that the majority of Lebanese nurses $(81.57 \%)$ were highly aware of IC measures which was a very good score compared to the literature, at the same time the study highlighted the IC topics where deficiencies (hand hygiene and blood borne diseases) were found and stressed on areas for improvements. As for nurses' trainings, the study showed that Lebanese hospitals were implementing continuous and regular IC training programs for their nurses that covered most of the important IC topics. Also the study showed that nurses who had a high awareness in IC measures, educated more their patients and family members on respiratory hygiene, reason for isolation and usage of PPE, and as per patients and family members' statement the JCI accredited hospital appeared to be the most hospital in educating its patients on HAI $(58.3 \%)$ and in providing brochures regarding hand hygiene and / or respiratory hygiene $(81.7 \%)$, this was explained by the presence of Standard PCI.11, in JCI standards (fifth edition) that requires the hospital to educate its patients and family members on IC measures and to encourage them to participate in the implementation and use of infection prevention and control practices in the hospital.

\section{Recommendations:}

Although 99.1\% of Lebanese nurses were trained on IC measures, the study recommended for the hospitals to put more efforts on their trainings for all HCW (especially nurses) on hand hygiene being one of the most important measures in preventing HAI and since only $44.2 \%$ of nurses were aware of hand hygiene as mentioned in the results, also training shall include all other IC measures (isolation, usage of PPE, and standard 
precautions etc...). These trainings should be obligatory and done regularly and continuously to all $\mathrm{HCW}$ to increase their awareness of IC, in addition to improve $\mathrm{HCW}$ practices toward IC measures. The recommendations were also for hospitals to perform regular audits by authorized personnel to closely monitor HCW compliance with IC practices, to put more emphasis on engaging the patients and family members in the process of care and to educate them on IC measures, and to perform audits to monitor this process of education. And finally to enhance IC culture of partnership between patients, their families and HCPs. As for the MoPH, and since the JCI accredited hospital showed the highest level of education on infection control measures $(58.3 \%)$, so the recommendations were to include a new standard in the Lebanese accreditation standards, that requires patient and family members' education on IC measures in mainly HAI and their risks, hand hygiene, respiratory hygiene, reason for isolation and usage of PPE, and to provide them with brochures for hand hygiene and their indications, also the standard must mention encouraging the patients and families to participate in the implementation and the use of IC practices, so that all Lebanese hospitals will be requested to educate their patients and families on these IC topics during their hospital stay. For future researchers the recommendations were to include more hospitals, to include a wide range of HCWs not only nurses, and to explore the reasons behind the non-education of patients and their families on IC measures.

Table 1: Nurses' Awareness of Various IC Topics.

\begin{tabular}{llll}
\hline Variable & N & $\%$ \\
\hline
\end{tabular}

HAI

Aware

138.0

$63.6 \%$

Not Aware

79.0

$36.4 \%$

Total

217.0

$100.0 \%$

Handling of biomedical waste

Aware

206.0

$94.9 \%$

Not Aware

11.0

$5.1 \%$

Total

217.0

$100.0 \%$

Handling of infected linen

Aware

211.0

$97.2 \%$

Not Aware

6.0

$2.8 \%$

Total

217.0

$100.0 \%$ 


\section{Handling / disposal of sharps}

Aware

Not aware

189.0

$87.1 \%$

Total

\section{Hand Hygiene}

Aware

96.0

$44.2 \%$

Not aware

121.0

$55.8 \%$

Total

217.0

$100.0 \%$

\section{Blood borne diseases}

Aware

30.0

$13.8 \%$

Not Aware

187.0

$86.2 \%$

Total

217.0

$100.0 \%$

\section{Additional safety measures}

Aware

Not aware

118.0

$54.4 \%$

Total

99.0

$45.6 \%$

217.0

$100.0 \%$

\section{Personal Protective Equipment (PPEs)}

Aware

156.0

$71.9 \%$

Not aware

61.0

$28.1 \%$

Total

217.0

$100.0 \%$

Table 2: IC Awareness of Nurses by their Demographic Characteristics.

\section{High awareness \# Low awareness \\ P- Value}
$\mathrm{N}(\%)$
$\mathrm{N}(\%)$

Nurses' Gender

\section{Male \\ Female}

Total

Nurses' Age

$$
\begin{aligned}
& \text { 20-25 years } \\
& \text { 26-30 years } \\
& \text { 31-35 years } \\
& \text { 36-40 years } \\
& >40 \text { years }
\end{aligned}
$$

$$
\begin{array}{r}
68(81.9 \%) \\
109(81.3 \%) \\
177(81.6 \%)
\end{array}
$$

$15(18.1 \%)$

$25(18.7 \%)$

$40(18.4 \%)$

$\begin{array}{lrr}39(79.6 \%) & 10(20.4 \%) & 0.778 \\ 60(78.9 \%) & 16(21.1 \%) & \\ 46(85.2 \%) & 8(14.8 \%) & \\ 21(80.8 \%) & 5(19.2 \%) & \\ 11(91.7 \%) & 1(8.3 \%) & \end{array}$


Total

$177(81.6 \%)$

Unit

Medicine

$38(82.6 \%)$

$26(68.4 \%)$

$25(89.3 \%)$

$19(73.1 \%)$

$21(87.5 \%)$

$35(97.2 \%)$

Hemato-Onco

Pediatrics

Total

Nurses' Job Title

HN

$\mathrm{RN}$

PN

Total
$13(68.4 \%)$

$177(81.6 \%)$
$111(84.7 \%)$

$42(68.9 \%)$

$177(81.6 \%)$

$1(4 \%)$

0.004

$20(15.3 \%)$

$19(31.1 \%)$

$40(18.4 \%)$

Nurses' years of service at current hospital
$<1$ year
1-5 years
6-10 years
$>10$ years
Total

$16(76.2 \%)$

$5(23.8 \%)$

0.887

$67(80.7 \%)$

$16(19.3 \%)$

$59(83.1 \%)$

$12(16.9 \%)$

$35(83.3 \%)$

$7(16.7 \%)$

$177(81.6 \%)$

$40(18.4 \%)$

Educational level

TS or lower
BS/LT
Master
Total

$44(66.7 \%)$

$22(33.3 \%)$

0.001

101 (86.3\%)

$16(13.7 \%)$

$32(94.1 \%)$

$2(5.9 \%)$

$177(81.6 \%)$

$40(18.4 \%)$ 
Table 3: Nurses' Educational Activities for patients/family members by Nurses' level of IC Awareness.

\section{High awareness \# Low awareness P- Value}

$\mathrm{N}(\%) \quad \mathrm{N}(\%)$

IC measures

Educated

Did not educate

Total

$$
\begin{array}{r}
167(94.4 \%) \\
10(5.6 \%) \\
177(100 \%)
\end{array}
$$

$40(100 \%)$

Hand Hygiene

Educated

Did not educate

Total

Respiratory Hygiene

Educated

$107(64.1 \%)$

$16(42.1 \%)$

0.013

Did not educate

Total

$60(35.9 \%)$

$22(57.9 \%)$

$167(100 \%)$

$38(100 \%)$

Reason for isolation

Educated

Did not educate

Total

Usage of PPE

$$
\begin{aligned}
& \text { Educated } \\
& \text { Did not educate } \\
& \text { Total }
\end{aligned}
$$

HAI and the risk of acquiring a HAI

Educated

Did not educate

Total
$149(89.2 \%)$

$18(10.8 \%)$

$167(100 \%)$

$144(86.2 \%)$

$23(13.8 \%)$

$167(100 \%)$

$60(35.9 \%)$

$107(64.1 \%)$

$167(100 \%)$
$26(68.4 \%)$

0.001

$12(31.6 \%)$

$38(100 \%)$

$22(57.9 \%)$

0.000

$16(42.1 \%)$

38 (100\%)

$11(28.9 \%)$

0.414

$27(71.1 \%)$

38 (100\%) 


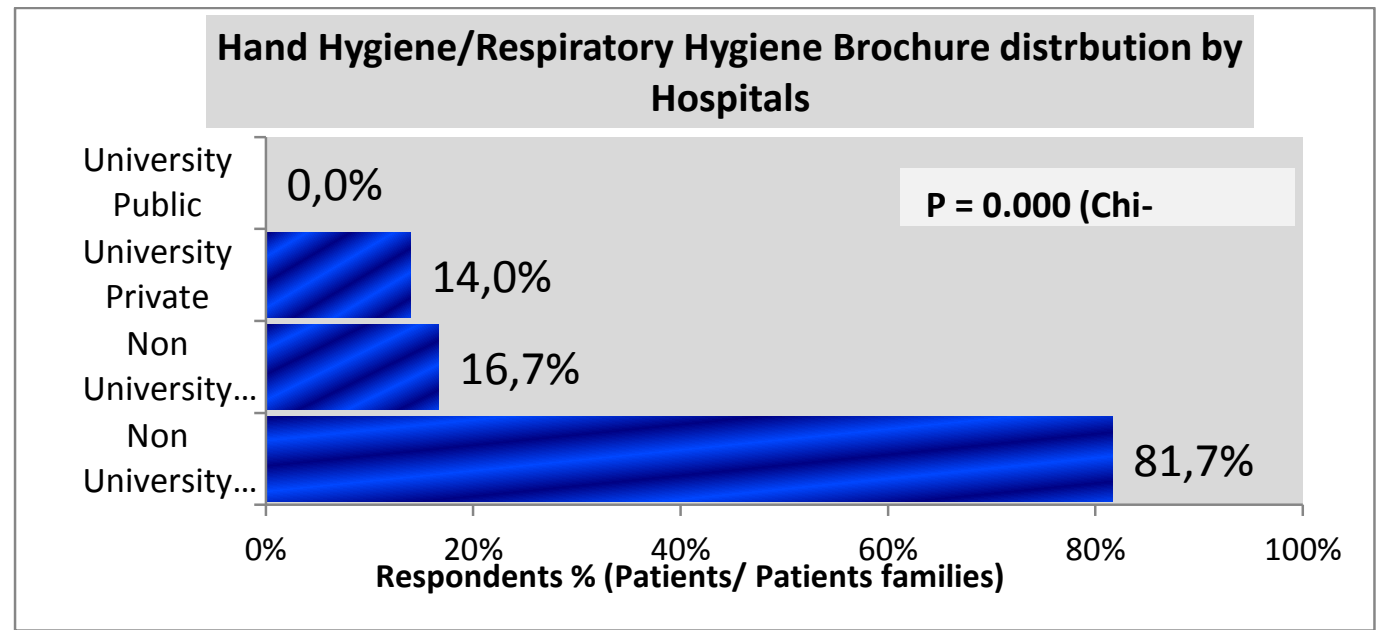

Figure 1: Hand Hygiene/Respiratory Hygiene Brochure distribution by Hospitals.

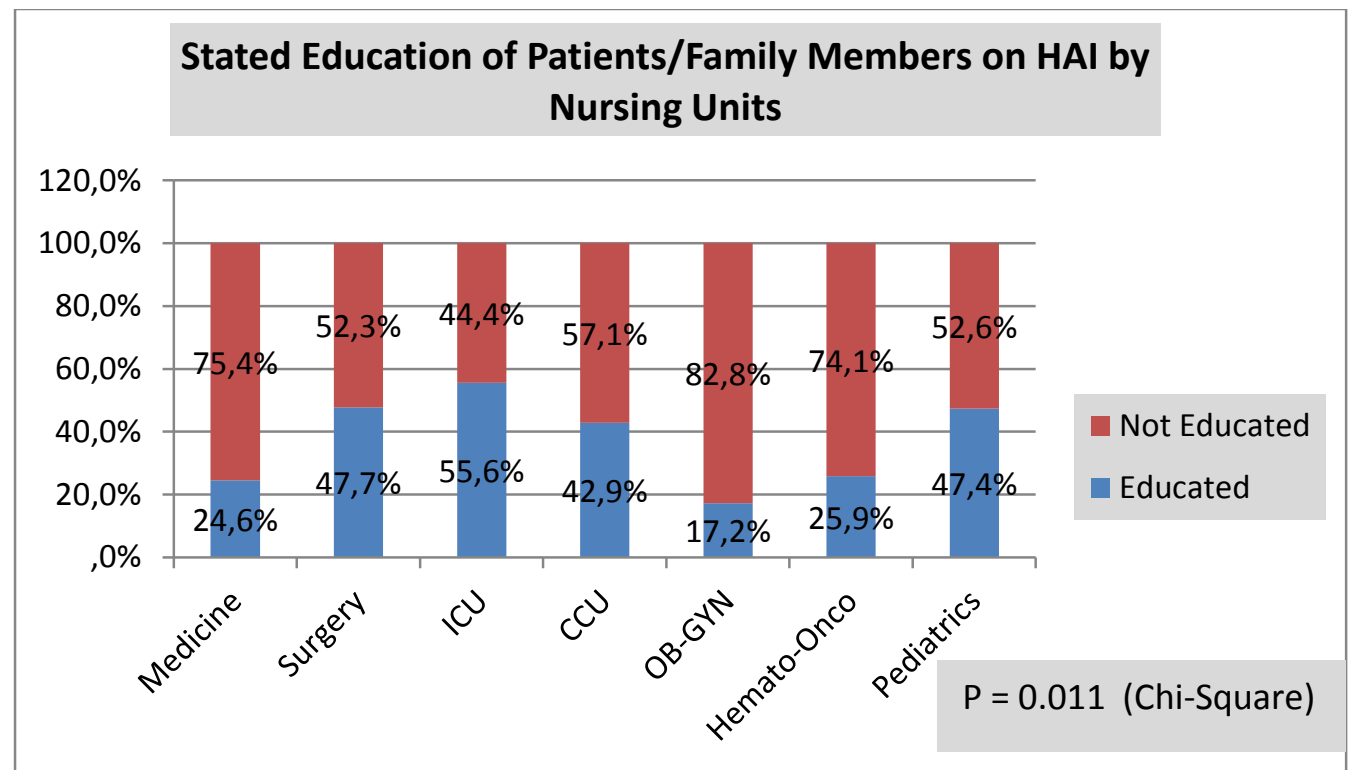

Figure 2: Stated Education of Patients/Family Members on HAI by Nursing Units.

\section{References:}

1. S. Mudedla, K. Reddy, M. Sowribala, W. Tej, "A Study on knowledge and awareness of standard precautions among Healthcare workers at Nizam's institute of medical sciences hyderabad," 2014.

2. "Agency for health care research and quality (AHRQ)," Agency for health care research and quality (AHRQ), April 2015. [Online]. Available: http://www.AHRQ.gov/. [Accessed 2015]. 
3. H. Seale, A. Chughtai, R. Kaur, P. Crowe, L. Phillipson, Y. Novytska, J. Travaglia, "Ask, speak up, and be proactive: Empowering patient infection control to prevent health care-acquired infections," American Journal of Infection Control, 2015.

4. K. Vaz, D. McGrowder, R. Alexander-Lindo, L. Gordon, L. Brown, R. Irving, "Knowledge, Awareness and Compliance with Universal Precautions among Health Care Workers at the University Hospital of West Indies, Jamaica," International Journal of Occupational and Environmental Medicine, vol. 1, 2010.

5. R. Dixon, "Control of Healthcare Associated Infections, 1961-2011," Center for Disease Control and Prevention, 2011.

6. "Prevention of Hospital Acquired Infections," World Health Organization, 2002.

7. "Core Components for Infection Prevention and Control Program," World Health Organization (WHO), Switzerland, 2008.

8. "Joint Commission International," Joint Commission, 1 January 2016.

[Online].

Available: http://www.jointcommission.org/standardsinformation/spsgs.aspx.

9. JD. Siegel JD, E. Rhinehart, M. Jackson, L. Chiarello, "Center of Disease Control and Prevention," Healthcare Infection Control Practices Advisory Committee (HICPAC), 2007. [Online]. Available: http://www.cdc.gov/ncidod/dhgp/pdf/isolation2007.pdf.

10. T. LAnders, S. Abusalem, M. Coty, J. Bingham, "Patient-centered hand hygiene: The next step in infection prevention," American Journal of Infection Control, 2012.

11. C. Regina, A. Molassiotis, C. Eunice, C. Virene, H. Bedcy, L. ChitYing, L. Pauline, S. Frances, Y. Ivy, "Nurses' Knowledge of and compliance with universal precautions in an acute care hospital," International Journal of Nursing Studies , pp. 157-163, 2002.

12. M. Askarian, K. Aramesh, C. John Palenik, "Knowledge, attitude and practice toward contact isolation precautions among medical students in Shiraz, Iran," American Journal of Infection Control, 2002.

13. A. Shah Salehi, P. Garner, "Occupational injury history and universal precautions awareness: a survey in Kabul hospital staff," Salehi and Garner BMC Infectious Disease, 2010.

14. A. A Charya, J. Khandekaar, A. Sharma, T. HR, A.Kataria, "Awareness and Practices of Standard Precautions for Infection Control among Nurses in a Tertiary Care Hospital," The Nursing Journal of India, 2013.

15. A. Elgilany, K. Badawy, B. Sarraf, "Knowledge of health care providers of standards precautions and Infection Control at Students' 
hospital, Mansoura University, Egypt," TAF Preventive Medicine Bulletin, 2012.

16. A. Tenna, E. Stenehjem, L. Margoles. E. Kacha. H. Blumberg, R. Kempker, "Infection Control Knowledge, Attitudes, and Practices among Healthcare Workers in Addis Ababa, Ethiopia," Infection Control Hospital Epidemiology, 2013.

17. S. ALZahrani, F. Al-Army, M. Ghonaim, O. Abo-Salem, "Awareness and Knowledge of Medical Students and Interns about Infection Control Measures," International Journal of Medical Sciences and Public Health, 2013.

18. P. Miller, B. Farr, "Survey of patients' knowledge of nosocomial infections," American Journal of Infection Control, 1989.

19. R. Abbate, G. Giuseppe, P. Marinelli, I. Angelill, "Patients' knowledge, attitudes and behavior toward hospital-associated infections in Italy," American Journal of Infection Control, 2008.

20. M. Macleo, L. Shields, E. Owen, "A pilot study to investigate patients reported knowledge, awareness and beliefs on Healthcareassociated-infections," American Journal of Infection Control, 2008.

21. A. Sengupta, C. Rand, T. Perl, A. Milstone, "Knowledge, Awareness and Attitudes Regarding Methicillin Resistant Staphylococcus Aureus among Caregivers of Hospitalized Children," National Institute of Health (NIH), 2010.

22. M. Atti, A. Tozzi, G. Ciliento, M. Pomponi, S. Rinaldi, M. Raponi, "Healthcare Workers' and Parents' perception of measures for improving adherence to hand-hygiene," BMC Public Health, 2011.

23. S. Pan, K. Tien, I. Hung, Y. Lin, Y. Yang, M. Yang, M. Wang, S. Chang, "Patient empowerment in hand hygiene program: Differing points of view between patients/family members and healthcare workers in Asian culture," American Journal of Infection Control, 2013.

24. I. Ocran, D. Tagoe, "Knowledge and attitude of healthcare workers and patients on healthcare associated infections in a regional hospital in Ghana," Pacific Journal of Tropical Disease, 2014.

25. H. Seale, Y. Novytska, J. Gallard, R. Kaur, "Examining Hospital Patients' Knowledge and Attitudes Toward Hospital-Acquired Infections and Their Participation in Infection Control," Infection Control \& Hospital Epidemiology, 2015.

26. P. Chittick, S. Koppisetty, L. Lombardo, A. Vadhavana, A. Solanki, K. Cumming, V. Agboto, C. Karl, J. Band, "Assessing patient and hospital-caregiver understanding of and satisfaction with the use of contact isolation," American Journal of Infection Control, 2016. 
27. F. Bellissimo-Rodrigues, D. Pires, W. Zingg, D. Pittet, "Role of parents in the promotion of hand hygiene in the pediatric setting: a systematic literature review," Journal of Hospital Infection, 2016.

28. Joint Commission International Standards for Hospitals, Fifth Edition, 2014.

29. W.Ammar, R.Wakim, I.Hajj, "Ministry of Public Health," 2007. [Online]. Available: www.moph.gov.lb. 